Revista Brasileira de Agricultura Irrigada v.11, nº.6, p. 1804 - 1812, 2017

ISSN 1982-7679 (On-line)

Fortaleza, CE, INOVAGRI - http://www.inovagri.org.br

DOI: $10.7127 /$ rbai.v11n600594

Protocolo 594.17 - 04/03/2017 Aprovado em 19/07/2017

\title{
DESENVOLVIMENTO INICIAL DO MAMOEIRO SOB DOSES DE CINZAS VEGETAIS E COBERTURA MORTA EM SISTEMA ORGÂNICO
}

\author{
José de Paula Firmiano de Sousa ${ }^{1}$, Paulo Gleisson Rodrigues de Sousa ${ }^{2}$, Luana Soares da \\ Silva $^{3}$, Patrícia Freitas Alcântara ${ }^{4}$, Carlos Pedro de Menezes Costa ${ }^{5}$, Raimundo Nonato \\ Tavora Costa ${ }^{6}$
}

\section{RESUMO}

Objetivando avaliar o desempenho no oitavo mês de cultivo do mamoeiro em uma unidade de experimentação, investigou-se a influência dos fatores de produção adubação com cinza vegetal e cobertura morta. Instalou-se uma unidade de experimentação em um delineamento em blocos ao acaso com parcelas subdivididas, constituídas por quatro tratamentos primários, dois secundários e cinco blocos. Os tratamentos primários, doses de cinza vegetal, corresponderam a $(0,50,100$ e $150 \%)$ da quantidade recomendada deste fator. Como fator secundário utilizouse bagana de carnaúba como cobertura morta, sendo um tratamento constituído pela aplicação de $\left(16 \mathrm{t} \mathrm{ha}^{-1)}\right.$ e um tratamento sem aplicação de bagana de carnaúba. Decorridos 210 dias após o plantio, analisaram-se as variáveis altura da planta e o diâmetro do caule, medido a 0,20 m da superfície do solo, além do número de frutos por planta. De acordo com os resultados encontrados foi possível concluir que o uso da bagana de carnaúba como cobertura morta no solo se mostrou como uma alternativa promissora para o incremento nas variaveis analisadas na cultura do mamoeiro nas condições edafoclimáticas em que a pesquisa foi realizada; $\mathrm{O}$ fornecimento de adubos naturais para suprir o requerimento nutricional dos cultivos raramente se combina em proporções ideais, necessitando de ajustes com outras fontes de adubos e um período prévio de preparação do solo para o cultivo em sistema de produção orgânico; O uso de insumos orgânicos à base de cinza vegetal e bagana de carnaúba consistem numa alternativa a a ser utilizada na agricultura familiar, contribuindo para a obtenção de produtos livres de agentes químicos.

Palavras-chave: Carica papaya L., Crescimento, Transição agroecológica.

\footnotetext{
${ }^{1}$ Especialista em Agroecologia, Universidade Federal do Ceará, e-mail: zefirmiano@yahoo.com.br ${ }^{2}$ Mestrando em Engenharia Agrícola, Universidade Federal do Ceará, e-mail: paulo.ufc.agro@gmail.com ${ }^{3}$ Mestranda em Engenharia Agrícola, Universidade Federal do Ceará, e-mail: luanasa19@hotmail.com ${ }^{4}$ Mestranda em Engenharia Agrícola, Universidade Federal do Ceará, e-mail: paty.alcantara07@hotmail.com ${ }^{5}$ Professor do Instituto Federal de Educação, Ciência e Tecnologia do Piauí, e-mail: carlos.pedromenezes@ifpi.edu.br

6Professor titular da Universidade Federal do Ceará, e-mail: rntcosta1509@gmail.com
} 


\title{
INITIAL DEVELOPMENT OF MAMMARY UNDER VEGETABLE ASHES AND DEAD COVERAGE IN ORGANIC SYSTEMS
}

\begin{abstract}
Aiming to evaluate the performance in the eighth month of cultivation of papaya in an experimental unit, we investigated the influence of the factors of production fertilization with vegetal ash and mulch. An experimental unit was set up in a randomized block design with subdivided plots, consisting of four primary treatments, two secondary and five blocks. The primary treatments, doses of vegetal ash, corresponded to (0, 50, 100 and 150\%) of the recommended amount of this factor. As a secondary factor, carnauba bagana was used as mulch, a treatment consisting of the application of $\left(16 \mathrm{t} \mathrm{ha}^{-1}\right)$ and a treatment without application of bagna of carnauba. After 210 days after planting, the variables plant height and stem diameter, measured at $0.20 \mathrm{~m}$ from the soil surface, were analyzed, in addition to the number of fruits per plant. According to the results, it was possible to conclude that the use of the carnauba bagana as mulch in the soil was shown as a promising alternative for the increase in the variables analyzed in the papaya crop under the edaphoclimatic conditions in which the research was carried out; The supply of natural fertilizers to meet the nutritional requirements of crops rarely combines in ideal proportions, necessitating adjustments with other sources of fertilizers and a previous period of preparation of the soil for cultivation in an organic production system; The use of organic inputs based on vegetable ash and carnauba bagana is an alternative to be used in family farming, contributing to the production of products free of chemical agents.
\end{abstract}

Keywords: Carica papaya L., Growth, Agroecological transition

\section{INTRODUÇÃO}

O mamão (Carica papaya L.), pertence à família Caricaceae, tendo origem na América tropical (BARBOSA, 2012). Consiste numa cultura que pode ser cultivada durante todo o ano em locais com temperatura média anual de $25^{\circ} \mathrm{C}$.

Barreto et al. (2010) destacam algumas características da cultura que facilitam seu cultivo, tais como: ampla densidade de plantas por hectare, rápido desenvolvimento, fácil propagação, produção durante todo o ano e alta produtividade. Mesmo com estas facilidades para cultivo do mamoeiro, sua produtividade pode aumentar com a melhoria das práticas agrícolas e na implantação de novos métodos de cultivo, viabilizando assim a obtenção de incrementos na qualidade e produção total de diversas espécies frutíferas (SOUZA et al., 2007; MARINHO et al., 2008).

Tem-se verificado uma crescente demanda de mercado por mamão originário de sistemas orgânicos, nos quais não se empregam agrotóxicos e adubos sintéticos. Nestes sistemas são adotadas medidas a fim de garantir a obtenção de produtividade competitiva, de modo que o mamão orgânico seja comercializado a preços populares, podendo atender a um número significativo de consumidores (MARTELLETO et al., 2008).

Resende e Resende Júnior (2011) destacam um sistema alternativo de produção de alimentos: a agricultura orgânica. A produção orgânica acarreta em avanços ambientais com maior preservação dos recursos naturais, assim também como um valor agregado nos produtos dos pequenos produtores que se utilizam desse sistema.

Sugerindo assim, a utilização de cobertura morta que funciona como uma esponja, aumentando a retenção de água e protegendo o solo da erosão e adversidades do clima. Colabora ainda no controle de plantas daninhas, na redução de perdas de nutrientes por lixiviação e, ao se decompor, auxilia na adubação e manutenção de temperaturas adequadas ao bom desenvolvimento da vida no solo (CORREA et al., 2003; SOUZA et al., 2012).

A disponibilidade de nutrientes, presença ou ausência de metais pesados, são algumas 
características químicas que viabilizam o uso de resíduos, como cinza de madeira, na agricultura, assim também como a origem dos materiais e os tratamentos a que estes são submetidos. A adição de resíduos orgânicos em sistemas de cultivo orgânico permite a obtenção de um produto com maior valor agregado (SOFIATTI et al., 2007).

No entanto são poucas as informações técnicas do uso de métodos alternativos que viabilize a produção principalmente ao pequeno produtor, e assim o estudo teve como objetivo avaliar o desempenho no oitavo mês de cultivo do mamoeiro irrigado em uma unidade de experimentação submetida aos fatores de adubação com cinza vegetal e utilização de cobertura morta.

\section{MATERIAL E MÉTODOS}

O experimento foi conduzido em área localizada à jusante do açude Pereira de Miranda, pertencente ao Departamento Nacional de Obras Contra as Secas - Dnocs, município de Pentecoste, Ceará, no período de janeiro de 2014 a fevereiro 2015. A área do experimento está localizada nas seguintes coordenadas $3^{\circ} 48^{\prime} 14.0^{\prime \prime} \mathrm{S} \quad 3^{\circ} 15^{\prime} 51.7^{\prime \prime} \mathrm{W}$ e altitude de $25 \mathrm{~m}$. O clima da região é semiárido, seco, existindo uma pequena temporada úmida, a qual permite o desenvolvimento da vegetação rasteira (Embrapa, 1999).

Os atributos físicos e químicos do solo na camada de 0 - 0,2 $\mathrm{m}$, foram determinados no Laboratório de Solos e Água do Departamento de Ciências do Solo, pertencente ao Centro de Ciência Agrárias da Universidade Federal do Ceará, sendo os atributos químicos, $\{\mathrm{P}(\mathrm{mg} \mathrm{kg}$ $\left.{ }^{1}\right) ; \mathrm{K}^{+}\left(\mathrm{cmol}_{\mathrm{c}} \mathrm{kg}^{-1}\right) ; \mathrm{Na}^{+}\left(\operatorname{cmol}_{\mathrm{c}} \mathrm{kg}^{-1}\right) ; \mathrm{Ca}^{2+}$ $\left(\right.$ cmolc kg $\left.^{-1}\right) ; \mathrm{Mg}^{2+}\left(\mathrm{cmol}_{\mathrm{c} \mathrm{kg}}{ }^{-1}\right) ; \mathrm{Al}^{3+}\left(\right.$ cmol $_{\mathrm{c}} \mathrm{kg}^{-}$ ${ }^{1}$ ); M.O. ( $\left.\mathrm{g} \mathrm{kg}^{-1}\right)$; C/N; pH; CE (dS m$\left.{ }^{-1}\right)$; PST: 37,00; 0,32; 0,13; 2,50; 2,50; 0,10; 7,97; 10,00; $6,00 ; 0,20 ; 2,00)\}$ respectivamente e os atributos físicos \{Areia fina $\left(\mathrm{g} \mathrm{kg}^{-1}\right)$; Areia grossa $\left(\mathrm{g} \mathrm{kg}^{-1}\right)$; Silte $\left(\mathrm{g} \mathrm{kg}^{-1}\right)$; Argila $\left(\mathrm{g} \mathrm{kg}^{-1}\right)$; Argila natural $\left(\mathrm{g} \mathrm{kg}^{-1}\right)$; Densidade solo $\left(\mathrm{g} \mathrm{cm}^{-3}\right)$; Umidade a 0,033 MPa; Umidade a 1,5 MPa: 706,00; 40,00; 175,00; 39,00; 39,00; 1,62; 9,57;
4,74) respectivamente, sendo a classe textural franco arenosa.

O delineamento experimental foi em blocos ao acaso com parcelas subdivididas, constando de quatro tratamentos primários, dois secundários e cinco repetições, totalizando assim 40 unidades experimentais com quatro plantas úteis cada uma.

Os tratamentos primários constaram dos níveis correspondentes a 0, 50, 100 e 150\% da quantidade de cinza vegetal recomendada, sendo a cinza vegetal produzida em forno de padaria. Como fator secundário utilizou-se bagana de carnaúba, sendo um tratamento constituído pela aplicação de $16 \mathrm{t} \mathrm{ha}^{-1}$ de cobertura morta (B1) e um tratamento (sem aplicação de cobertura morta - B0).

A dose de cinza correspondente a $100 \%$ da necessidade foi de $80 \mathrm{~g}$ de $\mathrm{K}_{2} \mathrm{O}$ por planta, representando um total de 1195 g de cinza vegetal no primeiro ciclo de produção, sendo que as aplicações foram fracionadas em cinco parcelas.

Antes do estabelecimento da cultura do mamoeiro no campo realizou-se a semeadura das leguminosas Calopogonium mucunoides e Pueraria phaseoloides com vistas à sua incorporação e, portanto, a uma maior disponibilidade de nutrientes, sobretudo nitrogênio.

A cultura do mamoeiro, cultivar (Tainung 01) do grupo Formosa foi semeada em bandejas de polietileno preta de 168 células no dia 08 de junho de 2014, utilizando-se uma mistura de argila, areia e esterco curtido de bovino nas proporções 1:1:1. A germinação ocorreu entre 09 e 12 dias após a semeadura.

As covas no espaçamento de 2,5 m x 2,5 $\mathrm{m}$ foram preparadas manualmente nas dimensões de $0,40 \mathrm{~m}$ x $0,40 \mathrm{~m}$ x $0,40 \mathrm{~m}$ e preenchidas com $32 \mathrm{~L}$ de esterco curtido de bovino e ovino e a mesma quantidade com solo da parte superficial retirado das próprias covas.

As características químicas do material utilizado na cova em proporção de $50 \%$ de esterco curtido e $50 \%$ de solo da camada superficial da cova estão contidas na Tabela 1. 
Tabela 1 - Características químicas do material utilizado na adubação de fundação

\begin{tabular}{cccccccccc}
\hline \multicolumn{4}{c}{ Macronutriente $\left(\mathrm{g} \mathrm{kg}^{-1}\right)$} & \multicolumn{4}{c}{ Micronutriente $\left(\mathrm{mg} \mathrm{kg}^{-1}\right)$} \\
\hline $\mathrm{N}$ & $\mathrm{P}$ & $\mathrm{P}_{2} \mathrm{O}_{5}$ & $\mathrm{~K}$ & $\mathrm{~K}_{2} \mathrm{O}$ & $\mathrm{Ca}$ & $\mathrm{Mg}$ & $\mathrm{Cu}$ & $\mathrm{Zn}$ & $\mathrm{Mn}$ \\
\hline 3,70 & 1,40 & 3,20 & 3,60 & 4,40 & 6,90 & 6,00 & 14,10 & 6,40 & 208,60 \\
\hline
\end{tabular}

O plantio definitivo em covas ocorreu no dia 03 de julho de 2014, ou seja, 25 dias após a semeadura, quando as mudas estavam com seis folhas definitivas. Neste período e durante dois dias, as plantas receberam exposição total ao sol para fins de adaptação.

O replantio se deu 06 dias após o plantio.

Semanalmente eram realizados tratos culturais, tais como retirada das plantas invasoras de forma manual na projeção da copa e com enxada, entre plantas e linhas. Aos 40 dias após o plantio aplicou-se a primeira adubação com cinza vegetal e a cobertura morta com bagana de carnaúba. Decorridos 85 dias da primeira aplicação realizou-se a segunda adubação com cinza vegetal. A terceira aplicação com cinza vegetal deu-se no dia 13 de janeiro de 2015, portanto decorridos 65 dias da segunda aplicação.

Utilizou-se um sistema de irrigação localizada do tipo microaspersão com um emissor para duas plantas operando a uma vazão de $112 \mathrm{~L} \mathrm{~h}^{-1}$ para uma pressão de serviço 15 mca, cuja linha principal era de tubos de PVC (diâmetro nominal de $50 \mathrm{~mm}$ ), com linhas laterais constituídas por mangueiras de polietileno (diâmetro nominal de $16 \mathrm{~mm}$ ).
O requerimento de água da cultura foi calculado para os diferentes meses e estádios da cultura conforme a Equação 1:

$$
\mathrm{Ti}=\frac{\text { ETo. Kc. Ap }}{\text { N.qe }}
$$

em que: Ti: tempo de irrigação, h; ETo: evapotranspiração de referência diária, $\mathrm{mm} ; \mathrm{Kc}$ : coeficiente de cultivo da cultura; Ap: área útil por planta, $\mathrm{m}^{2}$; qe: vazão do emissor, $\mathrm{Lh}^{-1} ; N$ : número de emissores por planta.

A evapotranspiração de referência (ETo) foi calculada através do método de PenmanMonteih/FAO (1991), utilizando o software Cropwat. Os dados de entrada para o cálculo de ETo foram obtidos de uma série histórica para o município de Pentecoste relativa ao período 1970-1998, de acordo com Cabral (2000), para os meses de (janeiro; fevereiro; março; abril; maio; junho; julho; agosto; setembro; outubro; novembro e dezembro: 6,$15 ; 5,33 ; 4,14 ; 4,14$; 4,$28 ; 4,61 ; 5,21 ; 6,85 ; 7,83 ; 7,97 ; 7,77 ; 7,27$ $\mathrm{mm}$ ) respectivamente.

Já os coeficientes de cultivo nos diversos estádios fenológicos foram obtidos por Montenegro, Bezerra e Lima (2004), conforme a Tabela 2.

Tabela 2 - Coeficientes de cultivo do mamoeiro nos diferentes estádios fenológicos

\begin{tabular}{lll}
\hline Dias após a semeadura & Estádios & Kc \\
\hline $84-107$ & Vegetativo & 0,64 \\
$115-260$ & Floração/Frutificação & 1,16 \\
$267-380$ & Floração/Frutificação/Maturação & 1,19 \\
\hline
\end{tabular}

Decorridos 210 dias do plantio, analisaram-se as variáveis de crescimento diâmetro do caule e altura da planta, além do número de frutos por planta. Os dados de altura de planta foram mensurados em metros e o diâmetro do caule foram medidos em centímetro a $0,20 \mathrm{~m}$ da superfície do solo.

As análises estatísticas das variáveis foram analisadas utilizando-se o software Assistat 7.7 (SILVA; AZEVEDO, 2016) e
Excel. Na análise da variância, os tratamentos foram comparados pelo teste F, sendo considerado um nível mínimo de significância de $5 \%$ de probabilidade. As comparações entre médias para o tratamento secundário foram realizadas pelo teste de Tukey em níveis de 5\% e $1 \%$ de probabilidade. 
Os valores médios de diâmetro do caule do mamoeiro, altura de planta e número de frutos em função dos tratamentos com cinza vegetal e bagana são apresentados na Tabela 3.

Tabela 3 - Resumo da ANOVA para (DC), (AP) e (NF) em função dos fatores cinza e cobertura morta com bagana de carnaúba. Umirim - CE. 2016.

\begin{tabular}{|c|c|c|c|c|}
\hline \multirow{2}{*}{ Fonte de variação } & \multirow{2}{*}{ GL } & \multicolumn{3}{|c|}{ Quadrado médio } \\
\hline & & DC & AP & NF \\
\hline Cinza (C) & 3 & 2,36485 ns & $0,00856 \mathrm{~ns}$ & $0,10929 \mathrm{~ns}$ \\
\hline Resíduo (C) & 12 & 1,13332 & 0,03460 & 0,08703 \\
\hline Cobertura morta (CM) & 1 & $52,69320^{* *}$ & $0,68382^{* *}$ & $18,56062^{* *}$ \\
\hline $\mathrm{C} \times \mathrm{CM}$ & 3 & 0,71750 ns & 0,03215 ns & 0,20117 ns \\
\hline Resíduo (CM) & 16 & 1,83873 & 0,03399 & 0,11589 \\
\hline CV (L) & (\%) & 10,27 & 12,00 & 25,01 \\
\hline $\mathrm{CV}(\mathrm{CM})$ & $(\%)$ & 13,76 & 11,90 & 28,86 \\
\hline
\end{tabular}

(**) Efeito significativo a 0,01 e $\left(^{*}\right)$ a 0,05 de probabilidade; (ns) não significativo a nível de 0,05 de probabilidade pelo teste $\mathrm{F}$

A análise de variância indicou efeito não significativo em nível de 5\% de significância para a (DC), (AP) e (NF) nos diferentes níveis de tratamentos com cinza vegetal, porém indicou efeito significativo ao nível de $1 \%$ para todas as variáveis ao fator cobertura morta. A interação entre os fatores de produção (C e CM) também não foi significativa em nível de $5 \%$ de significância.

Embora não significativo estatisticamente para o nível de $5 \%$ de significância, as médias ajustadas para os tratamentos com a aplicação de cinza, sinalizam uma relação do efeito crescente para as variáveis, cujo a melhor resposta apresentada foi para o tratamento C2B1 com um diâmetro do caule de $11,81 \mathrm{~cm}$, valor esse $9,06 \%$ maior que o tratamento C0B1 e 28,28\% superior ao tratamento C3B0, menor resultado obtido em todos os tratamentos sem a aplicação de cobertura morta. Percebe-se ainda que todos os valores obtidos com a aplicação de cobertura morta foram superiores aos tratamentos sem a aplicação de cobertura morta, conforme pode se verificar na Figura 1.

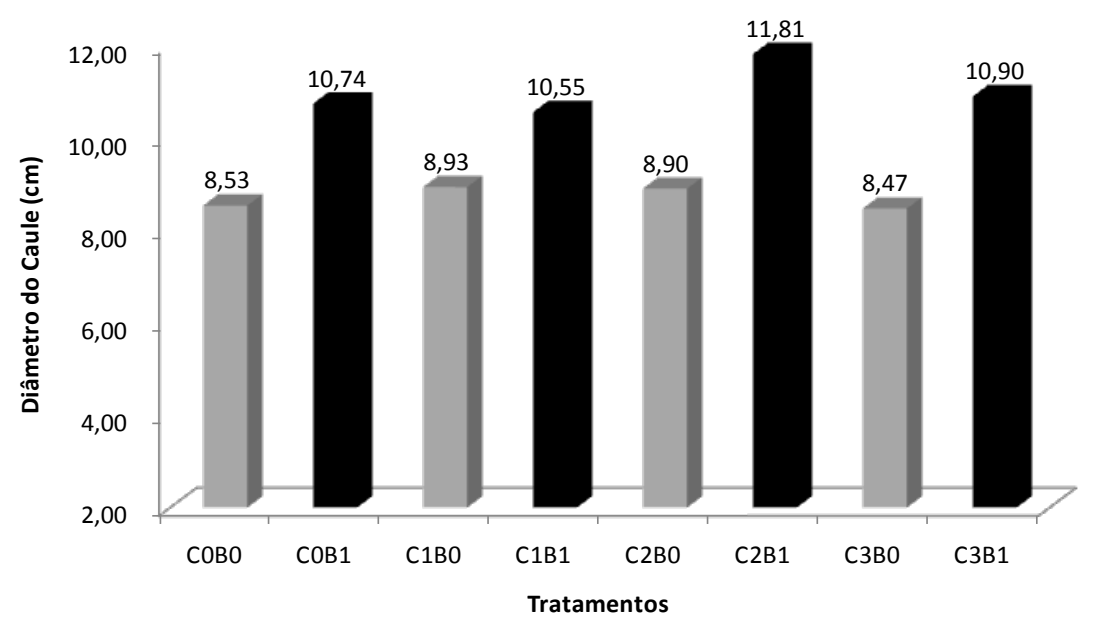

Figura 1. Diâmetro do caule para os diferentes tratamentos com cinza e bagana

De acordo com Silva et al. (2014) a variável de crescimento diâmetro do caule, constitui um importante parâmetro de correlação com a produção da cultura, sendo que plantas de mamoeiro com maior diâmetro do caule normalmente resultam em plantas mais produtivas em virtude da alta correlação genética entre essas características.

Oliveira et al. (2003) afirmam que o uso de bagana constitui uma importante fonte de 
retenção de umidade no solo, favorecendo as reações químicas e a disponibilidade dos nutrientes, pois muito embora a lâmina de água aplicada tenha sido a mesma para todos os tratamentos, a condição de solo protegido proporciona uma redução na evaporação de água diretamente do solo, além de criar um microclima favorável ao sistema radicular e, portanto, uma maior disponibilidade de nutrientes.

Além de todos esses benefícios gerados, a cobertura retém a água no solo mantendo-o mais tempo úmido e, assim, possibilita a aumentar os intervalos entre irrigações, no entanto, para que a utilização da cobertura seja viável faz-se necessário que novas alternativas de cobertura, disponíveis na região de cultivo, sejam avaliadas (BIZARI et al., 2009; FARIAS et al., 2015).

A melhor resposta apresentada para altura de planta do mamoeiro em função dos fatores "com e sem" cobertura morta foi para o tratamento C2B1 sendo esse de $1,75 \mathrm{~m}$, onde o mesmo encontra-se $10,28 \%$ superior ao menor resultado com cobertura morta C1B1 e 22,28\% superior ao tratamento C3B0, menor resultado obtido em todos os tratamentos sem a aplicação de cobertura morta. Logo assim, percebe-se que o efeito dos fatores para essa variável foi semelhante ao da variável diâmetro do caule, conforme pode se verificar na Figura 2.

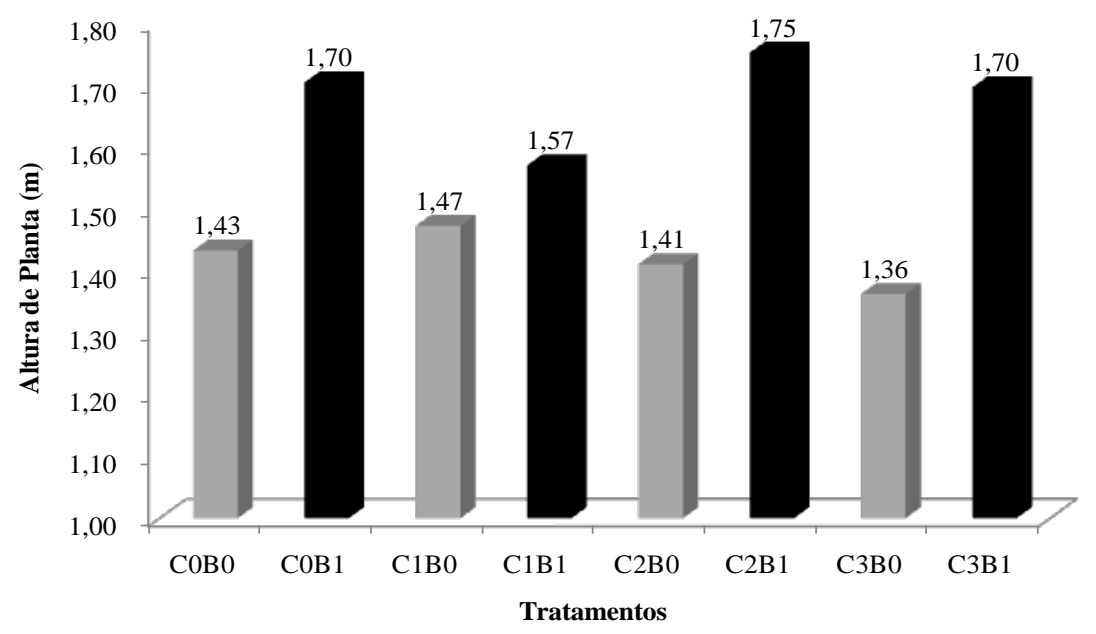

Figura 2. Altura de planta para os diferentes tratamentos com cinza e bagana

Ferreira et al. (2012) avaliando as correlações entre características morfoagronômicas de acessos de mamoeiro, verificaram correlação negativa ou inversa entre as variáveis altura de planta e produção. Já Silva et al. (2007), avaliando a correlações genotípicas de características morfoagronômicas no mamoeiro e implicações para o melhoramento genético, não verificaram correlação genotípica significativa entre altura de planta e número de frutos por planta.

Martelleto et al. (2008) avaliando cultivo orgânico do mamoeiro 'baixinho de santa Amália’ em diferentes ambientes de proteção, afirmam que o desenvolvimento do ciclo do mamoeiro foi marcadamente influenciado pelo tipo de ambiente de cultivo, sendo que o crescimento em altura, após 12 meses de transplantio das mudas, todas as diferenças entre médias de tratamentos foram significativas. Os autores constaram aumentos da ordem de $27,50 \%$ e $21 \%$ na altura das plantas, nas estruturas cobertas com o plástico, respectivamente, estufa e estufa sombreada, em comparação ao ambiente natural.

Conforme se observa na Figura 3, os valores médios referentes ao número de frutos por planta seguem o mesmo padrão das variáveis discutidas anteriormente, porém mostrando maior sensibilidade nos tratamentos sem a aplicação de cobertura, apontando esse como um insumo de importância no manejo da cultura para o desenvolvimento inicial em sistema orgânico de produção. 


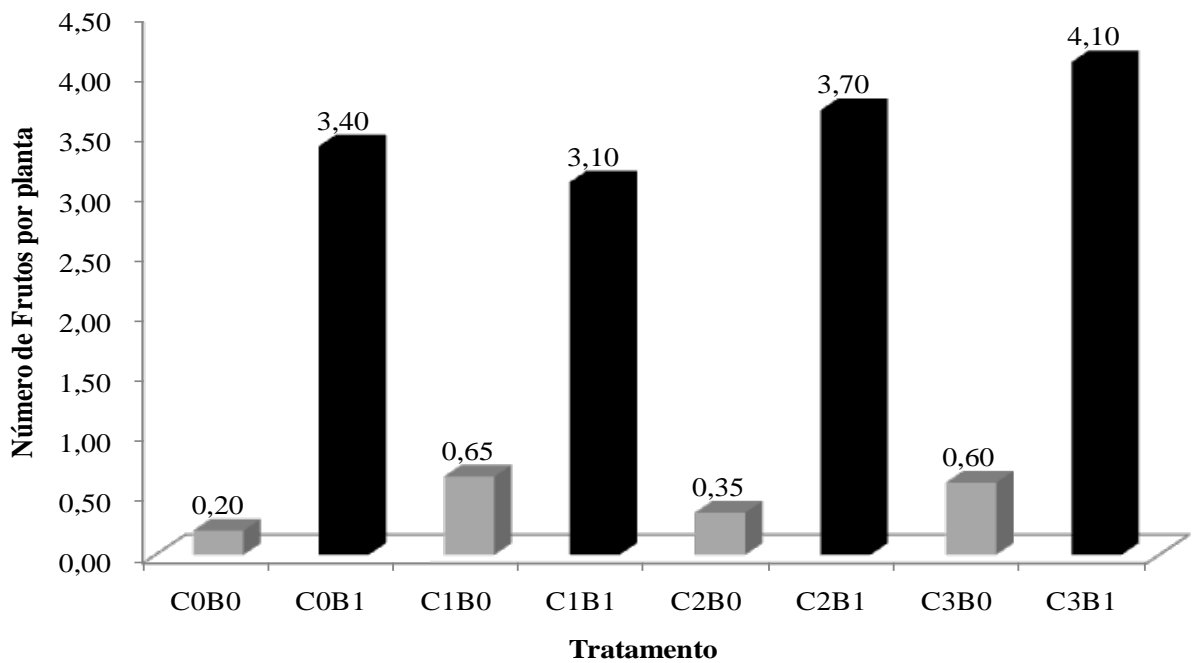

Figura 3. Número de frutos por planta para os diferentes tratamentos com cinza e bagana.

Oliveira et al. (2010) destacam que as características número de frutos por axila, largura da folha, altura da planta, número de flores por pedúnculo, comprimento de fruto e acidez são as principais determinantes das variações no número de frutos por planta.

Anjos et al. (2015) avaliando a fertilidade do solo, crescimento e qualidade de frutos do mamoeiro Tainung sob fertirrigação com potássio, constataram que o número de frutos por planta, massa média dos frutos e a produtividade se ajustaram ao modelo linear crescente.

Desse modo Shimazaki et al. (2007) afirmam que o aumento do número de frutos por planta está relacionado com a disponibilidade de potássio para planta, já que o potássio possui várias funções no metabolismo vegetal, como ativador enzimático, regulador da abertura estomática, fotossíntese, transporte de carboidratos e na respiração. Sendo que Viana et al. (2008), contataram relação crescente do número de frutos por planta em função do aumento das doses de potássio em um experimento com mamão formosa.

\section{CONCLUSÕES}

O uso da bagana de carnaúba como cobertura morta no solo se mostrou como uma alternativa promissora para o incremento nas variaveis analisadas na cultura do mamoeiro nas condições edafoclimáticas em que a pesquisa foi realizada;

O fornecimento de adubos naturais para suprir o requerimento nutricional dos cultivos raramente se combina em proporções ideais, necessitando de ajustes com outras fontes de adubos e um período prévio de preparação do solo para o cultivo em sistema de produção orgânico;

O uso de insumos orgânicos à base de cinza vegetal e bagana de carnaúba consistem numa alternativa a a ser utilizada na agricultura familiar, contribuindo para a obtenção de produtos livres de agentes químicos.

\section{REFERÊNCIAS}

ANJOS, D. C.; HERNANDEZ, F. F. F.; COSTA, J. M. C.; CABALLERO, S. S. U.; MOREIRA, V. O. G. Fertilidade do solo, crescimento e qualidade de frutos do mamoeiro Tainung sob fertirrigação com potássio. Revista Ciência Agronômica, v. 46, n. 4, p. 774-785, 2015.

BARBOSA, J. Q. Conservação pós-colheita de mamão 'sunrise solo' com uso de Quitosana. 2012. 50 f. Dissertação (Mestrado em Agronomia) Universidade Federal do Acre, Rio Branco, Rio Branco.

BARRETO, H. B. F.; COSTA, E. M.; OLIVEIRA, D. M. ; SILVA, K. B.; ARAUJO, J. A. M. Custos de produção e rentabilidade do 
cultivo do mamão formosa (Tainung $\mathrm{n}^{0} 1$ ) na cidade de Baraúna - RN. Revista Verde de Agroecologia e Desenvolvimento Sustentável, Mossoró, v. 5, n. 2, p. 96-102, 2010.

BARRETO, H. F. M. Impacto do manejo agroecológico da caatinga em unidades de produção familiar no oeste potiguar. 2010. 143 f. Dissertação (Mestrado em Ciência Animal) - Universidade Federal Rural do Semiárido. Mossoró.

CABRAL, R. C. Evapotranspiração de referência de Hargreaves (1974) corrigida pelo método Penman-Monteith/FAO (1991) para o estado do Ceará. 2000. $83 \mathrm{f}$. Dissertação (Mestrado em Irrigação e Drenagem) - Universidade Federal do Ceará. Fortaleza.

CORREA, T. M.; PALUDO, S. K.; RESENDE, F. V.; OLIVEIRA, P. S. R. Adubação química e cobertura morta em alho proveniente de cultura de tecidos. Horticultura Brasileira, v. 21, n. 4, p. 601-604, 2003.

DANTAS, J. L. L.; LIMA, J. F. Seleção e recomendação de variedades de mamoeiro avaliação de linhagens e híbridos. Revista Brasileira de Fruticultura, v. 23, n. 3, p. 617621, 2001.

FERREIRA, J. P.; SCHMILDT, O.; SCHIMILDT, E. R.; PIANTAVINHA, W. C.; CATTANEO, L. F. Correlações entre características morfo-agronômicas de acessos de mamoeiro. Enciclopédia Biosfera, v. 8, n. 14, p. 246-257, 2012.

MARINHO, A. B.; BERNARDO, S.; SOUSA, E. F.; PEREIRA, M. G.; MONNERAT, P. H. Produtividade e qualidade de frutos de mamão cultivar 'golden' sob diferentes lâminas de irrigação e doses de potássio no norte de Espírito Santo. Engenharia Agrícola, v. 28, n. 3, p. 417-426, 2008.

MARTELLETO, L. A. P.; RIBEIRO, R. L. D.; SUDOMARTELETO, M.; VASCONCELLOS, M. A. S.; MARIN, S. L.
D.; PEREIRA, M. B. Cultivo orgânico do mamoeiro 'Baixinho de Santa Amália' em diferentes ambientes de proteção. Revista Brasileira de Fruticultura, v. 30, n. 3, p.662666, 2008.

MONTENEGRO, A. A. T.; BEZERRA, F. M. L.; LIMA, R. N. Evapotranspiração e coeficientes de cultura do mamoeiro para a região litorânea do Ceará. Engenharia Agrícola, v. 24, n 2, p. 464 - 472, 2004.

OLIVEIRA, C. A. P; SOUZA, C. M. Influência da cobertura morta na umidade, incidência de plantas daninhas e de broca-do-rizoma (cosmopolites sordidus) em um pomar de bananeiras (musa spp.). Revista Brasileira de Fruticultura, v. 25, n. 2, p. 345-347, 2003.

OLIVEIRA, E. J.; LIMA, D. S.; LUCENA, R. S.; MOTTA, T. B. N.; DANTAS, J. L. L. Correlações genéticas e análise de trilha para número de frutos comerciais por planta em mamoeiro. Pesquisa Agropecuária Brasileira, v. 45, n. 8. p. 855-862, 2010.

RESENDE, S. A. A.; RESENDE JÚNIOR, J. C. Cultivo orgânico: origem, evolução e importância socioeconômica e ambiental. Enciclopédia Biosfera, v. 7, n. 13, p. 1119 1127, 2011.

SHIMAZAKI, K. I.; DOI, M.; ASSMANN, S. M.; KINOSHITA, T. Regulação da luz do movimento estomático. Annual Review of Plant Biology, v. 58, p. 219-247, 2007.

SILVA, F. F.; PEREIRA, M. G.; RAMOS, H. C. C.; DAMASCENO JUNIOR, P. C.; PEREIRA, T. N. S.; IDE, C. D. Correlações genotípicas de características morfoagronômicas no mamoeiro e implicações para o melhoramento genético. Crop Breeding and Applied Biotechnology, v. 7, p. 345-352, 2007.

SILVA, J.; ALMEIDA, C. L. S.; DANTAS, J. L. L.; SILVA, J. R.; TORRES, J. F. Caracterização agronômica de novos genótipos de mamoeiro na chapada do Apodi. In: Congresso Brasileiro de 
Fruticultura, 23. 2014, Cuiabá. Fruticultura: oportunidades e desafios para o Brasil, 2014.

SOFIATTI, V.; LIMA, R. L. S.; GOLDFARB, M.; BELTRÃO, N. E. M. Cinza de madeira e lodo de esgoto como fonte de nutrientes para o crescimento do algodoeiro. Revista de Biologia e Ciências da Terra, v. 7, n 1, p. 144152, 2007.

SOUZA, A. A.; SANTOS, P. C. T.; BEZERRA, O. M. P. A. Agroecologia. Centro Colaborador em Alimentação e Nutrição do Escolar (CECANE/UFOP), Universidade Federal de Ouro Preto, Ouro Preto, 2012.

SOUZA, T. V.; PAZ, V. P. S.; COELHO, E. F.; PEREIRA, F. A. C.; LEDO, C. A. S.
Crescimento e produtividade do mamoeiro fertirrigado com diferentes combinações de fontes nitrogenadas. Irriga, v. 12, n. 4, p. 563574, 2007.

SILVA, F. de A. S.; AZEVEDO, C. A. V. The Assistat Software Version 7.7 and its use in the analysis of experimental data. African Journal of Agricultural Research, v. 11, n. 39, p. 3733-3740, 2016.

VIANA, T. V. A.; SANTOS, F. S. S.; COSTA, S. C.; AZEVEDO, B. M.; SOUSA, A. E. Diferentes doses de potássio, na forma de nitrato de potássio, aplicadas via fertirrigação no mamão formosa. Revista Ciência Agronômica, v. 39, n. 1, p. 34-38, 2008. 